\title{
Fragilidad y deterioro cognitivo asociados al estado nutricional en personas mayores institucionalizadas
}

\author{
L. Regueiro-Folgueira ${ }^{\mathrm{a}}$, A. Maseda ${ }^{\mathrm{a}}$, L. Lorenzo-López ${ }^{\mathrm{a}}$, C. Diego-Diez ${ }^{\mathrm{b}}$, D. \\ Facal $^{\mathrm{c}}$, J.C. Millán-Calenti ${ }^{\mathrm{a}}$ \\ ${ }^{a}$ Universidade da Coruña, Grupo de Investigación en Gerontología, Instituto de Investigación Biomédica de \\ A Coruña (INIBIC), Complexo Hospitalario Universitario de A Coruña (CHUAC), SERGAS, A Coruña, \\ España \\ ${ }^{b}$ Complejo Gerontológico La Milagrosa, Asociación Provincial de Pensionistas y Jubilados (UDP) de A \\ Coruña, A Coruña, España \\ ${ }^{c}$ Universidade de Santiago de Compostela, Departamento de Psicoloxía Evolutiva e da Educación, Santiago \\ de Compostela, La Coruña, España
}

Objetivos: Fragilidad y deterioro cognitivo son situaciones que afectan con frecuencia a las personas mayores institucionalizadas, estando estrechamente relacionadas con el estado nutricional. El objetivo de este trabajo fue determinar la asociación del fenotipo de fragilidad y el deterioro cognitivo, así como su distribución en función del estado nutricional en personas mayores institucionalizadas.

Método: Se realizó un estudio transversal con una muestra de 157 personas de 65 o más años institucionalizadas en un complejo gerontológico. Se realizó una valoración geriátrica integral que incluía, entre otras variables, la valoración del estado nutricional mediante el Mini Nutritional Assesment (MNA), el fenotipo de fragilidad mediante criterios de Fried y el estado cognitivo mediante el Mini Mental State Examination (MMSE) o el Severe MMSE (SMMSE).

Resultados: La media de edad fue de 84,83 \pm 8,02 años. En cuanto al estado nutricional, la mayoría de los sujetos estaban en riesgo de malnutrición $(57,3 \%)$, seguidos de un estado nutricional adecuado $(28,7 \%) \mathrm{y}$, en menor porcentaje, de los malnutridos $(14,0 \%)$. La prevalencia de fragilidad supuso un $69,7 \%$, y el $78,7 \%$ de la muestra presentaba deterioro cognitivo. De acuerdo al estado nutricional, encontramos que de las personas con un estado nutricional normal, el $42,2 \%$ eran frágiles y el 52,3\% presentaban deterioro cognitivo. En el grupo de riesgo nutricional, el $77,8 \%$ eran frágiles y el $87,1 \%$ tenían deterioro cognitivo. Por último, en el caso de los sujetos malnutridos, el $95 \%$ presentaron fragilidad y todos, el 100\%, tenían deterioro cognitivo. Se observó, además, una correlación negativa entre la puntuación obtenida en el MNA y la puntuación en el MMSE/SMMSE y el número de ítems referidos al síndrome de fragilidad.

Conclusiones: El estado nutricional se encuentra estrechamente relacionado con el síndrome de fragilidad y el deterioro cognitivo entre las personas mayores de 65 o más años institucionalizadas. Las intervenciones dirigidas a un mejor estado nutricional deberían considerar todos los factores que pueden afectar a su deterioro.

Este trabajo ha sido financiado por la Xunta de Galicia (ED431C 2017/49 y Redes FrailNet IN607C 2016/08) y REGIDEM IN607C 2017/02. 